\title{
Active core rewarming avoids bioelectrical impedance changes in postanesthetic patients
}

\author{
Alma Rebeca Gutiérrez-Cruz ${ }^{1}$, Bernardo Soto-Rivera', Bertha Alicia León-Chávez² ${ }^{2}$ Ernesto Suaste-Gómez ${ }^{3}$, \\ Daniel Martinez-Fong ${ }^{4}$, Juan Antonio González-Barrios ${ }^{5^{*}}$
}

\begin{abstract}
Background: Postoperative hypothermia is a common cause of complications in patients who underwent laparoscopic cholecystectomy. Hypothermia is known to elicit electrophysiological, biochemical, and cellular alterations thus leading to changes in the active and passive membrane properties. These changes might influence the bioelectrical impedance (BI). Our aim was to determine whether the BI depends on the core temperature.
\end{abstract}

Methods: We studied 60 patients (52 female and 8 male) age 40 to 80 years with an ASA I-II classification that had undergone laparoscopic cholecystectomy under balanced inhalation anesthesia. The experimental group $(n=30)$ received active core rewarming during the transanesthetic and postanesthesic periods. The control group $(n=30)$ received passive external rewarming. The BI was recorded by using a 4-contact electrode system to collect dual sets of measurements in the deltoid muscle. The body temperature, hemodynamic variables, respiratory rate, blood-gas levels, biochemical parameters, and shivering were also measured. The Mann-Whitney unpaired $t$-test was used to determine the differences in shivering between each group at each measurement period.

Measurements of body temperature, hemodynamics variables, respiratory rate, and BI were analyzed using the twoway repeated-measures ANOVA.

Results: The gradual decrease in the body temperature was followed by the $\mathrm{Bl}$ increase over time. The highest $\mathrm{Bl}$ values $\left(95 \pm 11 \Omega\right.$ ) appeared when the lowest values of the temperature $\left(35.5 \pm 0.5^{\circ} \mathrm{C}\right)$ were reached. The active core rewarming kept the body temperature within the physiological range (over $36.5^{\circ} \mathrm{C}$ ). This effect was accompanied by low stable values ( $68 \pm 3 \Omega$ ) of Bl. A significant decrease over time in the hemodynamic values, respiratory rate, and shivering was seen in the active core-rewarming group when compared with the controls. The temporal course of shivering was different from those of body temperatue and BI. The control patients showed a significant increase in the serum-potassium levels, which were not seen in the active-core rewarming group.

Conclusions: The Bl analysis changed as a function of the changes of core temperature and independently of the shivering. In addition, our results support the beneficial use of active core rewarming to prevent accidental hypothermia.

\section{Background}

The postoperative hypothermia is a morbid condition that frequently affects patients recovering from general anesthesia [1]. Although the etiology of postoperative hypothermia is still under debate, it has long been associated with impaired metabolism, negative nitrogen balance, injury severity, hemorrhage, and multiple organ failure [2-4]. The occurrence of those

\footnotetext{
* Correspondence: jantgonzalez@issste.gob.mx

5Laboratorio de Medicina Genómica, Hospital Regional "Primero de Octubre",

Av. IPN, No. 1669, Mexico, D. F., C.P. 07760, Mexico

Full list of author information is available at the end of the article
}

dysfunctions depends on endogenous factors (endogenous hypothermia) or accidental factors (unintentional hypothermia) $[4,5]$. Postoperative hypothermia, in turn, may lead to severe complications such as negative catabolism and nitrogen balance, reduced resistance to infections, delayed wound healing, impaired coagulation, myocardial ischemia, and cardiac morbidities [6-8]. In particular, the cardiac complication may result in an overall higher mortality especially in patients with coronary artery disease [9]. Physiologically, the thermal input is integrated at numerous levels within the nervous system, but the

\section{Biomed Central}


hypothalamus is the dominant controller [10]. The inhibition of the thermoregulatory center in the hypothalamus by general anesthetics and sedatives is one of the causes of postoperative hypothermia, via mechanisms not completely known [11]. In contrast, the consequence of postoperative hypothermia on electrical properties especially of brain, heart, and neuromuscular activity is more characterized. Clinical evidence shows that hypothermia decreases the passive cable properties (membrane resting potential and amplitude, resistance) and impairs neuromuscular activity in such a magnitude that it can be recorded by noninvasive electrophysiological techniques [12-14].

Traditionally, bioelectrical impedance (BI) has been used as a noninvasive method to assess body-fluid compartments $[15,16]$ and body composition [17]. The resistance, reactance, and impedance of each arm, leg, and trunk can be easily quantified by using hand and foot contact electrodes [18-20]. In the clinic, BI analysis has been successfully used to determine the cellular uncoupling triggered by an increase of intracellular $\mathrm{Ca} 2+$ andor dephosphorylation of connexins during ischemia [21]. The cellular uncoupling is an important factor for the highest incidence of ventricular arrhythmia and cardiac failure $[21,22]$. Because the ischemia and coagulopathy are complications of prolonged hypothermia, mainly in patients with trauma or coronary artery disease [23-26], the routine monitoring of $\mathrm{BI}$ in hypothermic postoperative patients would be invaluable to determine the adequate treatment. Recently, the active core rewarming has demonstrated benefits of rapid rewarming of critically injured patients [27]. Therefore, we used active core rewarming in patients who had undergone laparoscopic cholecystectomy under balanced inhalation anesthesia to determine whether BI depends on the core temperature.

\section{Methods}

The study included 60 patients (52 female and 8 male) age 40 to 80 years with an ASA I-II classification. All patients had laparoscopic cholecystectomy at the "Hospital Regional Primero de Octubre" (ISSSTE, Mexico City, Mexico) and were divided into two groups. The control group (30 patients) was treated with roomtemperature parenteral solutions and a cotton blanket, which is referred to as passive external rewarming. The experimental group (30 patients) was treated with warmed parenteral solutions $\left(38.5^{\circ} \mathrm{C}\right)$ and a cotton blanket, which is referred to as active core rewarming. This protocol was approved by the Institutional Review Board of "Hospital Regional Primero de Octubre" (ISSSTE, Mexico City, Mexico) and written, informed consent was obtained from all patients.

\section{Anesthesia Technique}

The anesthesia procedures were done using a Dameca siesta iTS anesthesia machine (Dameca A.S., Roedovre, Denmark). All patients were treated with balanced inhalation anesthesia and complementary medication as follows; a) In the premedication phase, Midazolam (benzodiazepine); b) in the induction phase, Atropine (anticholinergic), Fentanyl (opioid), Cisatracurium (neuromuscular relaxant), and Propofol (inductors); c) in the maintenance phase, Fentanyl and Sevoflurane (volatile anesthetic agent). The dosage of all drugs was based on the body weight. The hemodynamic variables, respiratory rate, blood-gas levels, and biochemical variables were continuously measured by using a Cardiocap TM/ 5 monitor (Datex-Ohmeda Corp. Helsinki, Finland). During the surgical procedure, the heart rate, blood pressure, electrocardiographic trace, arterial gasometry, breathing rate, and esophageal temperature were continuously monitored. The patients treated with passive external rewarming (control group) received room-temperature parenteral solutions $(1250 \pm 250 \mathrm{~mL})$ during the entire study. The group of patients treated with active core rewarming received their parenteral solutions $(1250 \pm 250 \mathrm{~mL})$ warmed to $38.2^{\circ} \mathrm{C}$ by using a fluid warmer (Hl-90-SP-115; Smiths Medical ASD; Rockland, MA, USA) during the trans- and postsurgical period. Additional rewarming by using a cotton blanket was provided to both groups during the postanesthesia recovery. Patients treated with passive external rewarming remained in the postanesthesia care unit longer.

\section{Physiological Evaluation}

During the trans- and postsurgical periods, homeostasis variables (blood pressure, arterial gasometry, hematic biometry, glycemia, and serum potassium levels) were evaluated three times. The first set of measurements was done before the surgical procedure; the second set, during the surgical procedure before the gallbladder was removed; and the third set, after 30 minutes of stay in the postanesthesia care unit. The body temperature (BT), respiratory rate, heart rate (HR), electrocardiography trace (EKG), mean arterial blood pressure (MABP), oxygen saturation $\left(\mathrm{sO}_{2}\right)$, arterial carbon dioxide tension $\left(\mathrm{PaCO}_{2}\right)$, and shivering were measured every 30 minutes for 2 hours during the stay in the postanesthesia care unit.

\section{Bioimpedance analysis}

The BI was recorded by using a 4-contact electrode system to collect dual sets of segmental impedance measurements in the deltoid muscle. This apparatus was designed by Dr. E. Suaste-Gomez, and made by the staff of the Bioelectronics Section at CINVESTAV (Mexico City, Mexico). A constant current $(0.8 \mathrm{~mA})$ was applied 
to the lateral electrodes and the voltage drop was measured by the central electrodes. All assessments were made at $50 \mathrm{kHz}$ with and $0.8 \mathrm{~mA}$ of constant skin current. The voltage output value was obtained by the addition of input impedance (Zo), as Zo $+\Delta \mathrm{Z}$ (delta $\mathrm{Z}$ ). The voltage output recorded in the two central electrodes was proportional to the input current and the impedance measured by the lateral electrode as a function of the distance between electrodes. BI measurements were made after the patient had rested 30 minutes in the postanesthesia care unit, which has a controlled temperature and relative humidity. The BI measurements were repeated every 30 minutes for 2 hours.

\section{Statistical analysis}

All values were expressed as the mean $(s)$. We determined the proper sample size (n) for BI measurements by using the equation for comparison of two means and the following values, $\alpha=0.05 ; \beta=0.20$ with $80 \%$ of statistical power; $\mathrm{Z} \alpha=1.645 ; \mathrm{Z} \beta=0.842 ; \mathrm{S}=30 \Omega ; \mathrm{d}=$ $20 \Omega$. The Mann-Whitney unpaired $t$-test was used to determine differences in the number of patients who developed shivering between each group at each measurement period. Measurements of heart rate, mean arterial blood pressure, respiratory rate, body temperature, and BI were repeated for each group over time. Their differences were analyzed using the two-way repeated-measures ANOVA. All the statistical analysis was made using the PSPPIRE software version 0.6.0 for Linux (GNU General Public License). The null hypothesis was rejected at $P<0.05$ level.

\section{Results}

The age, weight, height, gender ratio, ASA classification, and duration of surgery were not statistically different between the two tested groups.

\section{Effect of active core rewarming on body temperature and bioelectrical impedance}

As expected, the body temperature in the control group remained as low as $35.5 \pm 0.5^{\circ} \mathrm{C}$, whereas the temperature increased gradually from $36^{\circ} \mathrm{C}$ to $37^{\circ} \mathrm{C}$ in patients treated with active-core rewarming. The temperature rise started 30 min after the onset of the treatment (Figure 1A). The patients treated with active core rewarming showed BI values of $68 \pm 3 \Omega$, which remained stable up to the end of the testing and were significantly different to those of the patients treated with passive external rewarming (Figure 1B). In the latter group, the BI values increased from $76 \pm 6 \Omega$ to reach the highest values of $95 \pm 11 \Omega$ at 60 minutes postanesthesia and then decreased to $88 \pm$ $9 \Omega$ at the end of study period (Figure 1B). These values were $30 \%$ higher than those found in the group treated with active core rewarming.
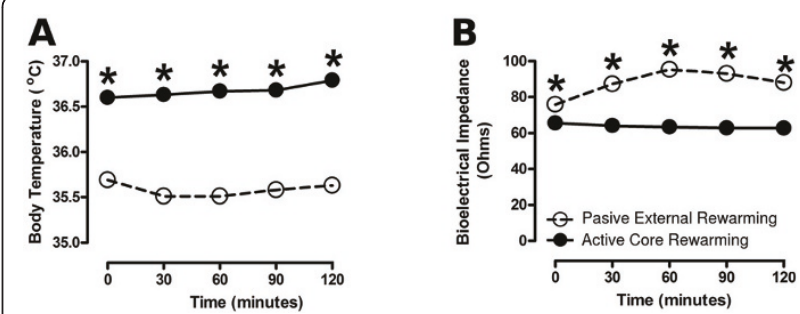

Figure 1 Effect of active core rewarming on body temperature and bioelectrical impedance of postoperative patients. Sixty adult patients underwent laparoscopic cholecystectomy under balanced inhalation anesthesia. A group of patients $(n=30)$ was treated with warmed parenteral solutions $\left(38.5^{\circ} \mathrm{C}\right)$ and a cotton blanket. This group is referred to as Active Core Rewarming $(\cdot)$. The other group $(n=30)$ was treated with room-temperature parenteral solutions and a cotton blanket. This group is referred to as Passive External Rewarming (o). The values are expressed as the mean (s). All $P$ values are $<0.001$.

\section{Effect of active core rewarming on physical and} biochemical variables

The percentage of control patients showing shivering was $25 \%$ at the beginning of the study, increased to $63 \%$ after $30 \mathrm{~min}$, and then gradually decreased to $25 \%$ up to the end of the study (Figure 2A). The counts of shivering frequency were significantly decreased (50\%) throughout the testing period in patients treated with active core rewarming when compared with those of the controls (Figure 2A). The heart rate of the postsurgical
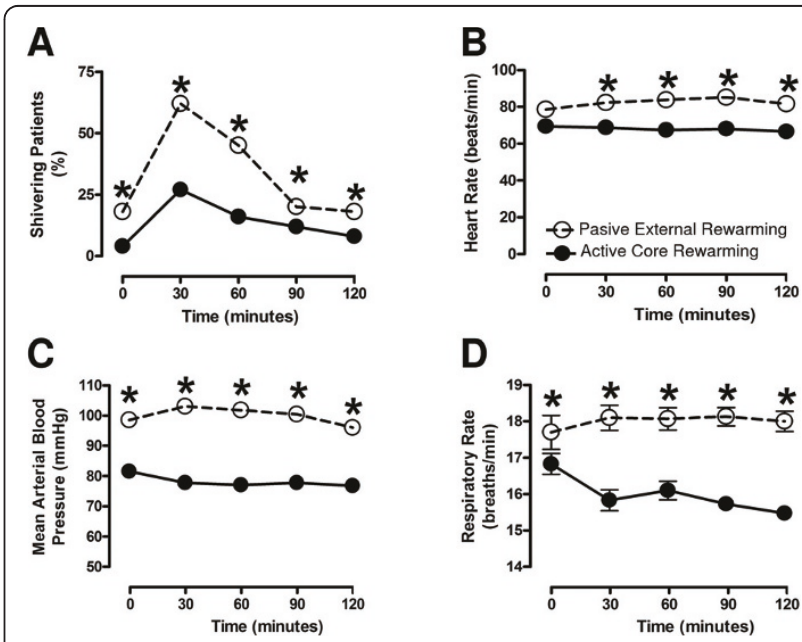

Figure 2 Effect of active core rewarming on physical variables of postoperative patients. Sixty adult patients underwent laparoscopic cholecystectomy under balanced inhalation anesthesia. A group of patients $(n=30)$ was treated with warmed parenteral solutions $\left(38.5^{\circ} \mathrm{C}\right)$ and a cotton blanket. This group is referred to as Active Core Rewarming $(\cdot)$. The other group $(n=30)$ was treated with room-temperature parenteral solutions and a cotton blanket. This group is referred to as Passive External Rewarming (o). The values are expressed as the mean (s). All $P$ values are $<0.001$ 
patients treated with active core rewarming remained stable at $67 \pm 1$ beats/min throughout the period of study. In contrast, the heart rate of patients who received passive external rewarming increased gradually from 77 to 85 beats/min (Figure 2B). A statistical difference was also found in the arterial blood pressure of patients treated with active core rewarming when compared with the controls. The arterial blood pressure of patients treated with active core rewarming was $20 \mathrm{mmHg}$ lower than the control (Figure 2C). The mean respiratory rate of patients treated with active core rewarming was statistically lower than the control values during the period of study (Figure 2D). No significant difference between groups was found in the measurements of $\mathrm{pH}$, oxygen or carbon dioxide tension, arterial-oxygen tension to the fractional inspired-oxygen ratio, arterial-oxygen saturation $(P>0.05)$, and in the biochemical variables (Table 1 ). The postoperative patients treated with passive external rewarming showed a significant increase in the serum-potassium levels during their stay in the anesthesia care unit (Table 1). The increase in the serum-potassium levels was not seen in the group treated with active core rewarming (Table 1).

\section{Discussion}

Our results show for the first time that the $\mathrm{BI}$ is directly affected by the changes of core temperature in postoperative patients subjected to laparoscopic cholecystectomy. We showed that the BI values are inversely associated with the changes in the body temperature, but not with the temporal course of shivering. The gradual decrease in the body temperature led to the increase in the BI values. The highest BI value $(95 \pm$ $11 \Omega$ ) appears at $60 \mathrm{~min}$ when the lowest temperature $\left(35.5 \pm 0.5^{\circ} \mathrm{C}\right)$ is reached. Furthermore, the active core rewarming maintained stable physiological values of body temperature (over $36.5^{\circ} \mathrm{C}$ ), which were associated with stable low values of the BI $(68 \pm 3 \Omega)$ over time. An increasing number of works show that the BI analysis is a reliable method to determine the fluid and body composition, and the cellular uncoupling in pathological conditions $[16,19,21]$. On this basis, we suggest that the increase in BI in postoperative hypothermic patients reflects the development of physiological, biochemical and cellular alterations. The precise molecular composition in fluid and body and the physiological mechanism underpinning BI changes in hypothermic patients remain to be determined.

Hypothermia is usually caused by a prolonged time of $\mathrm{CO}_{2}$ insufflation during the laparoscopic surgery [28]. In addition, the anesthetics, sedatives, and muscle relaxants are other factors able to potentiate the effect of $\mathrm{CO}_{2}$ insufflation on hypothermia during surgery [11]. These drugs are able to depress the hypothalamic thermoregulation and generate shivering as a compensatory mechanism [10]. In our study, the long surgical time $(82 \pm 5 \mathrm{~min})$ as compared with others [29] can account for the sustained hypothermia, the increased hemodynamic values, and the shivering observed in patients who received passive external rewarming. The increased

Table 1 Hemodynamic and Biochemical data during pre-, trans-, and postanesthetic periods in patients after laparoscopic cholecystectomy and treated with two different rewarming techniques

\begin{tabular}{|c|c|c|c|c|c|c|}
\hline \multirow[t]{2}{*}{ Variables } & \multicolumn{3}{|c|}{ Passive External Rewarming [mean (s), $n=30$ ] } & \multicolumn{3}{|c|}{ Active Core Rewarming [mean (s), $n=30$ ] } \\
\hline & Pre-anesthesia & Trans-anesthesia & Post-anesthesia & Pre-anesthesia & Trans-anesthesia & Post-anesthesia \\
\hline HR (beats/min) & $78.8 \pm 2.1$ & $64.5 \pm 1.9$ & $78 \pm 1^{*}$ & $79.2 \pm 2.1$ & $67.9 \pm 2$ & $71 \pm 1^{*}$ \\
\hline RR (breaths/min) & $15.1 \pm 0.4$ & $11.5 \pm 0.17$ & $16 \pm 0$ & $17.5 \pm 0.4$ & $11.6 \pm 0.2$ & $17 \pm 0$ \\
\hline $\mathrm{T}^{\circ} \mathrm{C}$ & $36.3 \pm 0.05$ & $35.9 \pm 0.06$ & $35 \pm 0$ & $36.38 \pm 0.07$ & $36.54 \pm 0.57$ & $36 \pm 0$ \\
\hline $\mathrm{SABP}(\mathrm{mmHg})$ & $133.9 \pm 3.5$ & $104.1 \pm 3.8$ & $126 \pm 2^{*}$ & $124.8 \pm 2.1$ & $110.1 \pm 3.1$ & $116 \pm 2^{*}$ \\
\hline DABP (mmHg) & $80.7 \pm 1.8$ & $64.0 \pm 1.9$ & $77 \pm 1$ & $76.4 \pm 1.8$ & $62.3 \pm 1.9$ & $69 \pm 1^{*}$ \\
\hline $\mathrm{sO}_{2}(\%)$ & $92.1 \pm 0.6$ & $98.4 \pm 0.1$ & $95 \pm 0.1$ & $92 \pm 0.52$ & $98.5 \pm 0.2$ & $96 \pm 0.1$ \\
\hline $\mathrm{PaCO}_{2}(\mathrm{mmHg})$ & $34 \pm 0.0$ & $30.3 \pm 0.6$ & $40.2 \pm 0.7$ & $35 \pm 0.4$ & $31 \pm 0.5$ & $41.1 \pm 0.4$ \\
\hline $\mathrm{PaO}_{2}(\mathrm{mmHg})$ & $64.6 \pm 1.4$ & $296.9 \pm 1.2$ & $76.1 \pm 1.5$ & $65.4 \pm 1.8$ & $322.9 \pm 9.1$ & $66 \pm 1.4$ \\
\hline $\mathrm{HCO}_{3}(\mathrm{mmol} / \mathrm{L})$ & $20.6 \pm 0.2$ & $17.78 \pm 0.5$ & $20.5 \pm 0.3$ & $21.6 \pm 0.2$ & $19.7 \pm 0.3$ & $21.13 \pm 0.21$ \\
\hline $\mathrm{Hgb}(\mathrm{mg} / \mathrm{dL})$ & $13.4 \pm 0.3$ & $12.8 \pm 0.3$ & $12.6 \pm 0.3$ & $13.5 \pm 0.3$ & $13.1 \pm 0.3$ & $12.9 \pm 0.3$ \\
\hline Hct (\%) & $42.9 \pm 1$ & $41 \pm 1$ & $39.9 \pm 0.1$ & $42.6 \pm 0.3$ & $40.4 \pm 0.9$ & $40 \pm 1$ \\
\hline Leukocytes $\left(10^{3} / \mathrm{cm}^{3}\right)$ & $7.5 \pm 2.8$ & $7.3 \pm 2.8$ & $7.3 \pm 2.8$ & $7.9 \pm 2.8$ & $7.7 \pm 3.1$ & $7.8 \pm 2.7$ \\
\hline Platelets $\left(\mathrm{mm}^{3}\right)$ & $283567 \pm 13030$ & $274200 \pm 12495$ & $271200 \pm 12818$ & $326500 \pm 9088$ & $326467 \pm 9007$ & $326333 \pm 9028$ \\
\hline $\mathrm{pH}$ & $7.41 \pm 0.01$ & $7.35 \pm 0.01$ & $7.30 \pm 0.01$ & $7.38 \pm 0.01$ & $7.38 \pm 0.01$ & $7 \pm 0.01$ \\
\hline $\mathrm{K}(\mathrm{mEq} / \mathrm{L})$ & $3.57 \pm 0.04$ & $3.96 \pm 0.03^{*}$ & $4.08 \pm 0.03^{*}$ & $3.58 \pm 0.03$ & $3.51 \pm 0.03^{*}$ & $3.45 \pm 0.04^{*}$ \\
\hline Glucose (mg/dL) & $95.87 \pm 4.8$ & $109.4 \pm 5.4^{*}$ & $116.5 \pm 5.2$ & $93.7 \pm 3.5$ & $88.7 \pm 4.2^{*}$ & $79.8 \pm 2.7^{*}$ \\
\hline
\end{tabular}

Data are expressed as mean $(s){ }^{*}$ Significant differences between active-core rewarming and passive-external rewarming $(P<0.05)$. $\mathrm{HR}$, heart rate; $\mathrm{HCO}$, bicarbonate; $\mathrm{SABP}$, systolic arterial blood pressure; $\mathrm{DABP}$, diastolic blood pressure; $\mathrm{SO}_{2}$, oxygen saturation; $\mathrm{PaCO}_{2}$, arterial carbon dioxide tension; PaO $\mathrm{C}_{2}$, arterial oxygen tension; $\mathrm{RR}$; respiratory rate; $\mathrm{Hgb}$, hemoglobin; $\mathrm{Hct}$, hematocrit; $\mathrm{K}$, potassium; $\mathrm{T}^{\circ} \mathrm{C}$, body temperature in Celsius. 
values of hemodynamic variables in the hypothermic patients of this study could result from the adrenergic response to hypothermia, which has been supported by others [30,31]. It has long been known that the adrenergic response caused by the increased release of norepinephrine in response to cold causes vasoconstriction and secondary hypertension $[32,33]$. In our study, the postoperative patients treated with passive external rewarming also showed a mild but significant increase in the serum-potassium levels during the trans- and postanesthetic period. This alteration is possibly caused by an overstimulation of the neuromuscular junction and ectopic contractions in shivering patients. Similar results have been found in electroconvulsive therapy, in which there is a fast increase of plasmatic potassium levels [34].

In agreement with previous results [35], the active core rewarming used in this study caused stable values of body temperature near the physiological range (over $36.5^{\circ} \mathrm{C}$ ) along with a significant decrease in the hemodynamic values and shivering. These results suggest that the control of core temperature is able to prevent the adrenergic response and maintain the passive electrical properties within the physiological range. On this basis, we suggest that the $\mathrm{BI}$ changes depend on the core temperature changes. Because of this property, the BI may be a useful tool to monitor the recovery from hypothermia in postoperative patients, especially for those with cardiac antecedents [22]. In addition to routine monitoring, the BI data might lead to more timely interventions, resulting in clinical improvement and a shorter stay in the anesthesia care unit.

\section{Conclusions}

The BI changed as a function of the core temperature and independently of the involuntary muscle activity. The physiological and molecular mechanisms underpinning the BI changes in hypothermic postoperative patients require further studies. In addition, our results support the beneficial use of active core rewarming to prevent accidental hypothermia and changes in $\mathrm{BI}$.

\section{Consent}

Each patient for publication freely gave a written informed consent. A copy of the written consent is available to the Editor-in-Chief of this Journal.

\footnotetext{
Acknowledgements

The authors thankfully acknowledge the ISSSTE for the financial support. We thank the General Director of ISSSTE (Miguel Angel Yunes-Linares) and the Director of "Hospital Regional Primero de Octubre" (Enrique Núñez González) for providing us the clinical and laboratory facilities. The invaluable support by the anesthesiology staff is also acknowledged. Thanks to Dr. Ellis Glazier for editing of the English-language text.
}

\section{Author details}

'Departamento de Anestesia, Hospital Regional "Primero de Octubre", Av. IPN, No. 1669, Mexico, D. F., C.P. 07760, Mexico. ${ }^{2}$ Área de Bioquímica y Biología Molecular, Facultad de Ciencias Qumícas, BUAP, 14 sur y Av. San Claudio, 72570, Puebla, Pue., México. ${ }^{3}$ Departamento de Ingeniera Eléctrica, CINVESTAV, Av. IPN No. 2508, México D.F., C.P. 06760, México.

${ }^{4}$ Departamento de Fisiología, Biofísica y Neurociencias, CINVESTAV, Av. IPN No. 2508, México D.F., C.P. 06760, México. ${ }^{5}$ Laboratorio de Medicina Genómica, Hospital Regional "Primero de Octubre", Av. IPN, No. 1669 Mexico, D. F., C.P. 07760, Mexico

\section{Authors' contributions}

ARG made the anesthesia procedure, and participated in the collection and analysis of the data. BSR participated in the data collection and analysis. BALCH participated in the data analysis. ESG designed the 4-contact electrode system recorder. DMF was the scientific advisor and prepared the final version of this manuscript. JAGB participated in the design of the study, in the data analysis, conceived the paper, and prepared the figures, table, and the final version of this manuscript. All authors read and approved the final version of the manuscript.

\section{Competing interests}

The authors have no financial and personal relationships with other people or organizations that could inappropriately influence their work, all within 3 years of beginning the work submitted.

Received: 22 June 2010 Accepted: 16 February 2011 Published: 16 February 2011

\section{References}

1. Sessler DI: Mild perioperative hypothermia. N Engl J Med 1997 336:1730-1737.

2. Zaballos Bustingorri JM, Campos Suarez JM: [Non-therapeutic intraoperative hypothermia: prevention and treatment (part II)]. Rev Esp Anestesiol Reanim 2003, 50:197-208.

3. Seekamp A, van Griensven M, Hildebrandt F, Wahlers T, Tscherne H: Adenosine-triphosphate in trauma-related and elective hypothermia. J Trauma 1999, 47:673-683

4. Hildebrand F, Giannoudis PV, van Griensven M, Chawda M, Pape HC: Pathophysiologic changes and effects of hypothermia on outcome in elective surgery and trauma patients. Am J Surg 2004, 187:363-371.

5. Insler SR, Sessler DI: Perioperative thermoregulation and temperature monitoring. Anesthesiol Clin 2006, 24:823-837.

6. Doufas AG: Consequences of inadvertent perioperative hypothermia. Best Pract Res Clin Anaesthesiol 2003, 17:535-549.

7. Mathews S, Al Mulla A, Varghese PK, Radim K, Mumtaz S: Postanaesthetic shivering-a new look at tramadol. Anaesthesia 2002, 57:394-398.

8. Kurz A, Sessler DI, Narzt E, Bekar A, Lenhardt R, Huemer G, Lackner F: Postoperative hemodynamic and thermoregulatory consequences of intraoperative core hypothermia. J Clin Anesth 1995, 7:359-366.

9. Qadan M, Gardner SA, Vitale DS, Lominadze D, Joshua IG, Polk HC Jr: Hypothermia and surgery: immunologic mechanisms for current practice. Ann Surg 2009, 250:134-140.

10. Morrison SF, Nakamura K, Madden CJ: Central control of thermogenesis in mammals. Exp Physiol 2008, 93:773-797.

11. Diaz M, Becker DE: Thermoregulation: physiological and clinical considerations during sedation and general anesthesia. Anesth Prog 2010, 57:25-32.

12. Zouridakis G, Papanicolaou AC, Simos PG: Intraoperative neurophysiological monitoring. Part 2: Neurophysiological background. J Clin Eng 1997, 22:321-327.

13. Kochs E: Electrophysiological monitoring and mild hypothermia. J Neurosurg Anesthesiol 1995, 7:222-228.

14. Sessler DI, Rubinstein EH, Moayeri A: Physiologic responses to mild perianesthetic hypothermia in humans. Anesthesiology 1991, 75:594-610.

15. Bracco D, Berger MM, Revelly JP, Schutz Y, Frascarolo P, Chiolero R: Segmental bioelectrical impedance analysis to assess perioperative fluid changes. Crit Care Med 2000, 28:2390-2396.

16. Jaffrin MY, Morel $\mathrm{H}$ : Body fluid volumes measurements by impedance: $A$ review of bioimpedance spectroscopy (BIS) and bioimpedance analysis (BIA) methods. Med Eng Phys 2008, 30:1257-1269. 
17. El Dawlatly AA: Perioperative bioelectrical impedence analysis in neurosurgery. Middle East J Anesthesiol 2005, 18:575-581.

18. Cornish BH, Jacobs A, Thomas BJ, Ward LC: Optimizing electrode sites for segmental bioimpedance measurements. Physiol Meas 1999, 20:241-250.

19. Elia M, Fuller NJ, Hardingham CR, Graves M, Screaton N, Dixon AK, Ward LC: Modeling leg sections by bioelectrical impedance analysis, dual-energy X-ray absorptiometry, and anthropometry: assessing segmental muscle volume using magnetic resonance imaging as a reference. Ann N Y Acad Sci 2000, 904:298-305.

20. Pietrobelli A, Morini P, Battistini N, Chiumello G, Nunez C, Heymsfield SB: Appendicular skeletal muscle mass: prediction from multiple frequency segmental bioimpedance analysis. Eur J Clin Nutr 1998, 52:507-511.

21. Cascio WE, Yang H, Muller-Borer BJ, Johnson TA: Ischemia-induced arrhythmia: the role of connexins, gap junctions, and attendant changes in impulse propagation. J Electrocardiol 2005, 38:55-59.

22. Albert NM, Hail MD, Li J, Young JB: Equivalence of the bioimpedance and thermodilution methods in measuring cardiac output in hospitalized patients with advanced, decompensated chronic heart failure. Am J Crit Care 2004, 13:469-479.

23. Lapointe $L A$, Von Rueden $K T$ : Coagulopathies in trauma patients. AACN Clin Issues 2002, 13:192-203

24. Leslie K, Sessler DI: Perioperative hypothermia in the high-risk surgical patient. Best Pract Res Clin Anaesthesiol 2003, 17:485-498.

25. Horn EP, Torossian A: [Prevention of perioperative hypothermia]. Anasthesiol Intensivmed Notfallmed Schmerzther 2010, 45:160-167.

26. Frank SM, Fleisher LA, Breslow MJ, Higgins MS, Olson KF, Kelly S, Beattie C: Perioperative maintenance of normothermia reduces the incidence of morbid cardiac events. A randomized clinical trial. JAMA 1997, 277:1127-1134

27. Taylor EE, Carroll JP, Lovitt MA, Petrey LB, Gray PE, Mastropieri CJ, Foreman ML: Active intravascular rewarming for hypothermia associated with traumatic injury: early experience with a new technique. Proc (Bayl Univ Med Cent) 2008, 21:120-126.

28. Huntington TR, LeMaster CB: Laparoscopic hypothermia: heat loss from insufflation gas flow. Surg Laparosc Endosc 1997, 7:153-155.

29. Salam IM, Own A, Kareem NA, Hameed OA, Yak CJ, Zaki KA: Laparoscopic cholecystectomy in the Academy Medical Centre, Khartoum, Sudan. East Afr Med J 2005, 82:10-13.

30. Fleisher LA, Frank SM, Sessler DI, Cheng C, Matsukawa T, Vannier CA: Thermoregulation and heart rate variability. Clin Sci (Lond) 1996, 90:97-103.

31. Frank SM, Cattaneo CG, Wieneke-Brady MB, El Rahmany H, Gupta N, Lima JA, Goldstein DS: Threshold for adrenomedullary activation and increased cardiac work during mild core hypothermia. Clin Sci (Lond) 2002, 102:119-125.

32. Lamke LO, Lennquist S, Liljedahl SO, Wedin B: The influence of cold stress on catecholamine excretion and oxygen uptake of normal persons. Scand J Clin Lab Invest 1972, 30:57-62.

33. Wilkerson JE, Raven PB, Bolduan NW, Horvath SM: Adaptations in man's adrenal function in response to acute cold stress. J Appl Physiol 1974, 36:183-189.

34. Bali IM: The effect of modified electroconvulsive therapy on plasama potassium concentration. Br J Anaesth 1975, 47:398-401.

35. Hasankhani H, Mohammadi E, Moazzami F, Mokhtari M, Naghgizadh MM: The effects of intravenous fluids temperature on perioperative hemodynamic situation, post-operative shivering, and recovery in orthopaedic surgery. Can Oper Room Nurs J 2007, 25:20-27.

\section{Pre-publication history}

The pre-publication history for this paper can be accessed here: http://www.biomedcentral.com/1471-2253/11/2/prepub

doi:10.1186/1471-2253-11-2

Cite this article as: Gutiérrez-Cruz et al: Active core rewarming avoids bioelectrical impedance changes in postanesthetic patients. BMC Anesthesiology 2011 11:2 